\title{
Analysis Method for Inertial Particle Separator
}

\author{
Farooq Saeed* and Ahmad Z. Al-Garni亡 \\ King Fahd University of Petroleum and Minerals, Dhahran 31261, Saudi Arabia
}

DOI: $\underline{10.2514 / 1.20245}$

\begin{abstract}
This paper describes an analysis method for an inertial particle separator system modeled as a multi-element airfoil configuration. The analysis method is implemented in a numerical tool that is able to perform impingement analysis using spherical, nonspherical particles as well as water droplets for a range of Reynolds number $\left(10^{-4} \leq R e \leq 5 \times 10^{5}\right)$. A limitations of the analysis tool is that it lacks an appropriate particle rebound model for the treatment of particle-wall collisions. The usefulness of the analysis tool is its use in conjunction with a multipoint inverse design tool for the design of a multi-element airfoil based inertial particle separator system model in an inverse fashion as opposed to the direct design methods being employed currently for this task. With such a design and analysis tool at hand, the design space can be explored as well as tradeoff studies can be performed that can aid in the development of a more efficient design methodology for multi-element airfoil based inertial particle separator systems.
\end{abstract}

\begin{tabular}{|c|c|}
\hline & Nomenclature \\
\hline$C_{d}$ & $=$ particle drag coefficient \\
\hline$c$ & $=$ airfoil chord length \\
\hline$D_{\text {eq }}$ & $=\begin{array}{l}\text { equivolumetric or mean volumetric } \\
\text { diameter }\end{array}$ \\
\hline $\boldsymbol{F}_{a}$ & $=$ aerodynamic force \\
\hline $\boldsymbol{F}_{g}$ & $=$ gravitational force \\
\hline $\begin{array}{l}g \\
(i, k)\end{array}$ & $\begin{array}{l}=\text { gravitational acceleration constant } \\
=\text { unit direction vectors in wind } \\
\text { reference frame }\end{array}$ \\
\hline$\left(\boldsymbol{i}_{p}, \boldsymbol{k}_{p}\right)$ & $\begin{array}{l}=\text { unit direction vectors in body } \\
\text { reference frame }\end{array}$ \\
\hline $\boldsymbol{k}_{1}, \boldsymbol{k}_{2}, \boldsymbol{k}_{3}, \boldsymbol{k}_{4}$ & $\begin{aligned} &= \text { Runge-Kutta coefficients used to } \\
& \text { integrate the momentum equation }\end{aligned}$ \\
\hline$\left(l_{0}, n_{0}\right)$ & $=$ trajectory direction vector \\
\hline$\left(l_{1}, n_{1}\right)$ & $=$ airfoil panel plane direction vector \\
\hline$m_{p}$ & $=$ particle mass, $\rho_{p} V_{p}$ \\
\hline$n$ & $=$ surface normal vector \\
\hline$p$ & $=$ ambient pressure \\
\hline Re & $\begin{aligned}= & \text { Reynolds number based on particle } \\
& \text { diameter, } \rho_{a} D_{\mathrm{eq}} U / \mu_{a}\end{aligned}$ \\
\hline $\boldsymbol{r}_{p}$ & $=$ particle position \\
\hline $\boldsymbol{r}_{p, i}=\left(x_{p, i}, z_{p, i}\right)$ & $\begin{array}{l}=\text { particle current position during } \\
\text { trajectory integration }\end{array}$ \\
\hline $\boldsymbol{r}_{p, i+1}=\left(x_{p, i+1}, z_{p, i+1}\right)$ & $\begin{array}{l}=\text { particle new position during } \\
\text { trajectory integration }\end{array}$ \\
\hline$S$ & $\begin{array}{l}=\begin{array}{l}\text { particle surface projection on the } \vec{U} \\
\text { perpendicular plane }\end{array}\end{array}$ \\
\hline$S_{p}$ & $=$ particle surface area \\
\hline$s$ & $=\frac{\text { airfoil panel surface arc length, }}{\sqrt{\left(x_{2}-x_{1}\right)^{2}+\left(z_{2}-z_{1}\right)^{2}}}$ \\
\hline$t$ & $=$ time \\
\hline$t_{0}$ & $\begin{aligned}= & \text { trajectory parametric equation } \\
& \text { parameter }\end{aligned}$ \\
\hline$t_{1}, t_{2}$ & $\begin{aligned}= & \text { airfoil panel parametric equation } \\
& \text { parameters }\end{aligned}$ \\
\hline
\end{tabular}

Presented as Paper 0014 at the 44th ASME, Reno, NV, 9-12 January 2006; received 25 September 2005; revision received 15 March 2007; accepted for publication 16 April 2007 . Copyright $@ 2007$ by the authors. Published by the American Institute of Aeronautics and Astronautics, Inc., with permission. Copies of this paper may be made for personal or internal use, on condition that the copier pay the $\$ 10.00$ per-copy fee to the Copyright Clearance Center, Inc., 222 Rosewood Drive, Danvers, MA 01923; include the code $\$ 10.00$ in correspondence with the CCC.

*Assistant Professor, Aerospace Engineering Department, KFUPM, Mail Box 1637. Member AIAA.

${ }^{\dagger}$ Professor and Chairman, Aerospace Engineering Department, KFUPM, Mail Box 842. Senior Member AIAA.

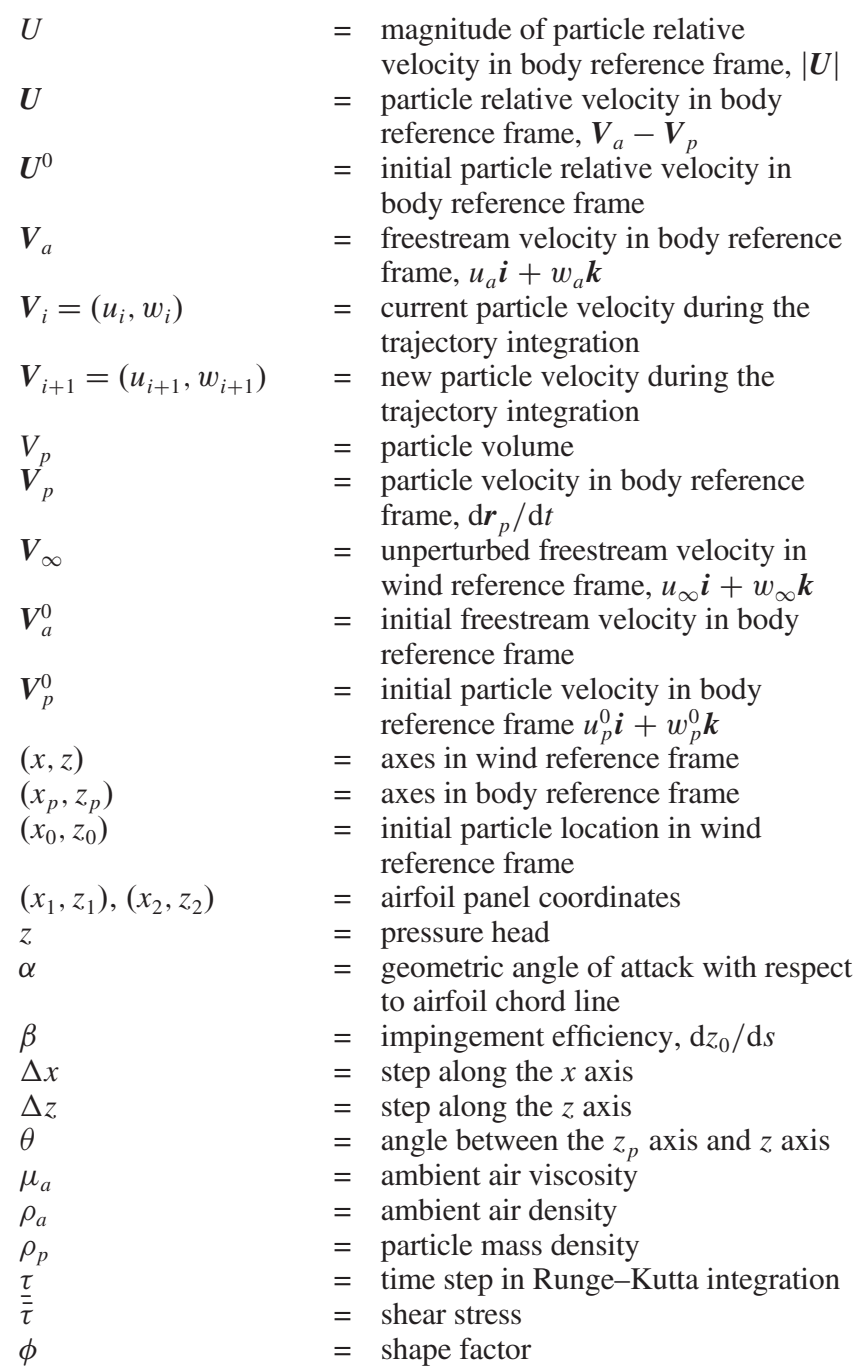

\section{Introduction}

T HE blades and vanes of a gas turbine engine are susceptible to erosion during prolonged flight operations in desertlike environments. This can result in a loss of power and hence limit the performance as well as life of the engine. The operating life of a helicopter engine operating in sandy environment can be as short as $50 \mathrm{~h}[\underline{1}, 2]$. In extreme cases, an engine can become inoperative with 


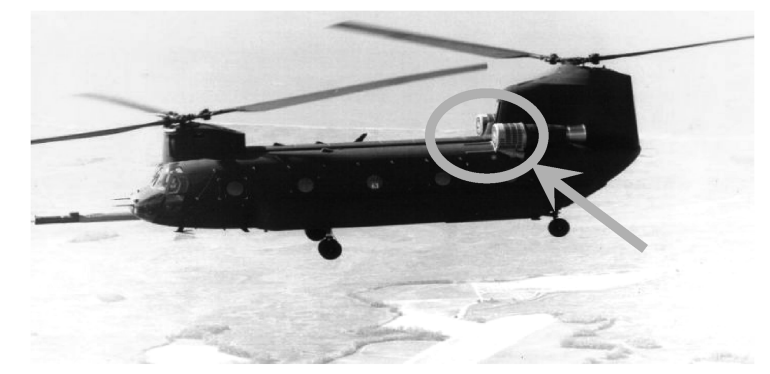

Fig. 1 Boeing CH-47D helicopter with an IPS system installed [ $\underline{3}$ ].

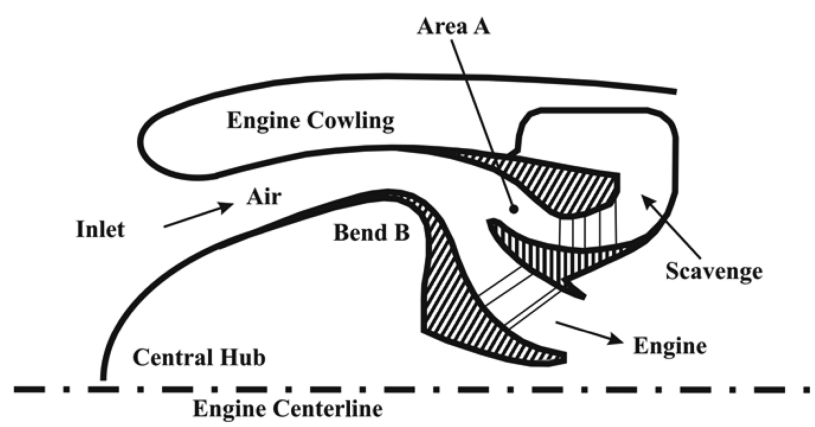

Fig. 2 Typical axisymmetric helicopter engine particle separator (adapted from [3]).

as little as half a pound of sand. Typically, coarse sand is found to result in the roughening of blade surfaces of axial compressors and turbines. In centrifugal compressors, wearing of the impeller leading and trailing edges at the root due to sand erosion often results in structural failure. Performance deterioration due to erosion can also lead to significant increase in specific fuel consumption [1]. It therefore becomes imperative that some form of protective device, such as an inertial particle separator (IPS) system, must be used on the engine inlets to prevent sand ingestion into the engine.

Currently there are two types of IPS systems in use: the swirl and the vaneless types. In the swirl type, vanes introduce a swirl to the contaminated inlet flow. The resulting centrifugal force causes the heavier sand particles to move over to the outer periphery and into a scavenge duct. The vaneless type relies on the specific contour of the inner walls of the inlet and the diffuser that direct the contaminants to the scavenge duct. To further enhance the separation of contaminants from the air, a hump imparts sufficient linear momentum to the contaminants that carries them into the scavenge area and the contaminant-free air enters the engine. Figure 1 [3] shows a Boeing $\mathrm{CH}-47 \mathrm{D}$ helicopter with the IPS system installed (shown inside the circle) on the engine inlets. The engine-mounted particle separator is an axisymmetric, bifurcated duct of the form shown in Fig. 2 (adapted from [3]). The different IPS systems available today are very similar in design. Contaminated air enters the device through the inlet annulus on the left, and around a sharp bend B. The bend is designed in such a manner that the inertia of the contaminants is sufficient to prevent them from following the air around the bend. Thus contaminants, such as sand, dust, etc., pass into a scavenge passage $\mathrm{A}$, and the contaminant-free clean air passes into the engine along the inner annulus.

The IPS system does a phenomenal job of keeping the engines clean and free from damage due to sand particles and other foreign

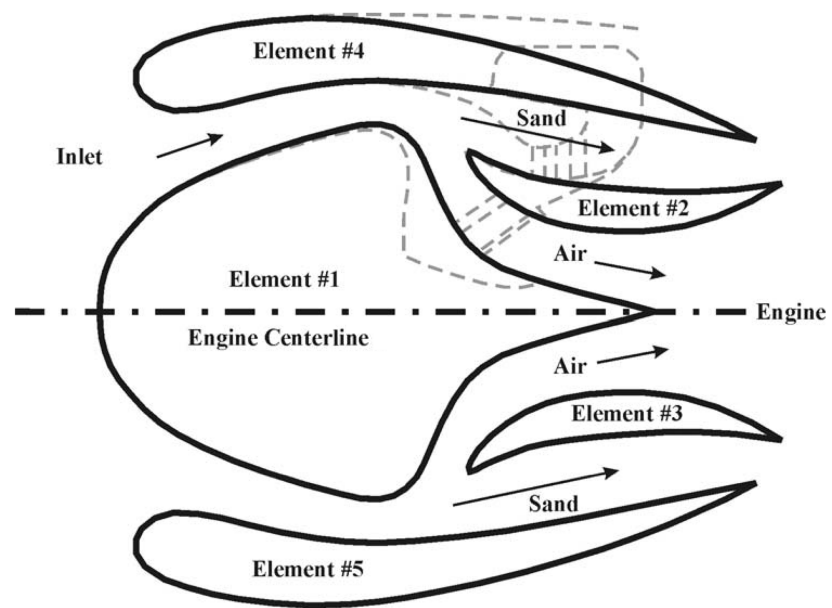

Fig. 3 A five-element airfoil configuration model for an IPS system.

object ingestion. Inertial particle separator systems (such as that shown in Fig. 2) are capable of moving large particles leaving the smaller ones to be trapped by the filters which greatly enhanced the life of the filter and offers maximum engine protection [3] . The main advantage is the large installed area required for such a system thus increasing the overall intake area. However, engines having IPS installed prevent the crew from conducting a thorough preflight of the engine inlet area. There is no single dust size, which can fully represent the range of sand and dust encountered in flight operations. Table 1 lists the typical types of sand referred to as standard test dust and their characteristics.

In literature, numerous methods for the analysis and design of an IPS system have been used [4-6] to improve the collection efficiency of such systems and achieve high levels of reliability and durability. The methods take advantage of advanced analytical and computational [computational fluid dynamics (CFD)] techniques for throughflow and particle trajectory analysis. Improvements in existing design are achieved through extensive analysis and experimental validation as in the case of [4]. Thus, the design is accomplished in a direct or hit-and-trial fashion and suggests that significant amounts of resources are required to accomplish the task. The analysis method presented in this paper is part of a greater effort to propose a new multipoint inverse design approach for the IPS systems as opposed to the hit-and-trial direct design approach. In a multipoint inverse design, the geometry is derived from a set of requirements and constraints on the flow as well as multiple design conditions thus making it a very powerful design approach. In this approach, it is assumed that the cross-sectional profile of the engine inlet with an integral IPS system can be treated as a multi-element airfoil configuration as illustrated in Fig. $\underline{3}$. A careful examination of Fig. $\underline{3}$ suggests that the path of sand particles can be directed by an appropriate design as well as positioning of airfoil elements 1-5. A preliminary look at the model suggest that proper positioning of elements 4 and 5 (forward and backward along the centerline chordwise direction or perpendicular to it) can help separate sand particles from being ingested into the engine and at the same time provide the required inlet mass flow rate to the engine. Moreover, a study into the design of the different elements along with their specific arrangements in a multi-element configuration can give insight into which airfoil arrangements and characteristics can prevent sand ingestion into the engine. In this process, the

Table 1 Types and characteristics of loose, dry sand $\left(\right.$ density $\left.=1422 \mathrm{~kg} / \mathrm{m}^{3}\right)$

\begin{tabular}{lcccc}
\hline \hline Sand types & Diameter range, $\mathrm{mm}$ & Diameter range, $\mu \mathrm{m}$ & Minimum volume, $\mathrm{m}^{3}$ & Mass, $\mathrm{kg}$ \\
\hline Very fine & $0.05-00.1$ & $50-100$ & $6.54 \mathrm{E}-14$ & $9.44 \mathrm{E}-11$ \\
Fine & $0.1-0.25$ & $100-250$ & $5.24 \mathrm{E}-13$ & $7.55 \mathrm{E}-10$ \\
Medium & $0.25-00.5$ & $250-500$ & $8.18 \mathrm{E}-12$ & $1.18 \mathrm{E}-08$ \\
Coarse & $0.5-1$ & $500-1000$ & $6.54 \mathrm{E}-11$ & $9.44 \mathrm{E}-08$ \\
Very coarse & $1-2$ & $1000-2000$ & $5.24 \mathrm{E}-10$ & $7.55 \mathrm{E}-07$ \\
\hline \hline
\end{tabular}


environment (sand particle distribution, etc.) and mass flow requirement (engine operational envelop) can be specified as design requirements. The design can then be accomplished by conducting tradeoff studies to optimize the location and profile of each of the elements.

The first step in this process is the development of an analysis method for a multi-element airfoil-type IPS system as shown in Fig. 3. In such a model, the flowfield must be determined first and used to determine the sand particle trajectories. The sand particle trajectories could then be used to find the impact or impingement regions (or surfaces) on all of the elements. The impingement characteristics together with the specific arrangement of the airfoil elements could serve as a guide or basis for developing a design methodology for such IPS systems. Currently, to our knowledge, no such design method exits to date. Hence, the work presented here is part of a larger effort to develop a novel design method for a multielement airfoil-type IPS system.

This paper presents the details of a mathematical model and the numerical solution technique developed for the analysis of a multielement airfoil-type IPS system to gain insight into the design of such systems. The analysis of such an IPS system can be divided into three main parts: 1) flowfield analysis, 2) trajectory analysis, and 3) impingement analysis. In the sections that follow, details of the flowfield, trajectory and impingement analyses, as well as the associated numerical techniques are presented. A validation example is presented to indicate the accuracy of the method. Then some results are presented to highlight the capabilities of the method. Suggestions for the improvement of the method are discussed in light of its strengths and limitations. A brief discussion on the design of the multi-element airfoil-type IPS system is also presented. Finally, the paper ends with some conclusions.

\section{Mathematical Models and Numerical Implementation}

\section{Flowfield Analysis}

To mathematically model the flow of air and particles through a typical IPS system, the IPS system is modeled as a multi-element airfoil configuration (five-element airfoil configuration shown in Fig. 3). Flow analysis of the five-element airfoil configuration is accomplished with the aid of an inviscid flow analysis method for multi-element configurations, such as the panel method of Hess and Smith [7]. The method determines the velocity potential field around a given multi-element airfoil configuration. Tuncer [7] presents the details of the panel method of Hess and Smith and, therefore, its discussion is left out of this paper. It is, however, noted here that the method [7] applies strictly to two-dimensional flows whereas the flow through the IPS system can be three dimensional depending upon the magnitude of swirl imparted to the flow within the IPS system. In this study, it is assumed that the magnitude of swirl velocity within the IPS system is small and that the flow can be treated as quasi two dimensional. Hence, the flow and trajectory analyses are carried out in a two-dimensional space $(x, z)$. In this study, two coordinate reference frames/axes are employed in the analyses: 1) body reference frame $\left(x_{p}, z_{p}\right)$ that is fixed to the particle (sand, water droplet, etc.) and used in the trajectory calculation, and 2 ) wind reference frame $(x, z)$ that is fixed to the multi-element airfoil configuration and is used for flowfield analysis about the given configuration. Figure 4 depicts the two reference coordinates frames/ axes as used in the analyses. The following transformation relation

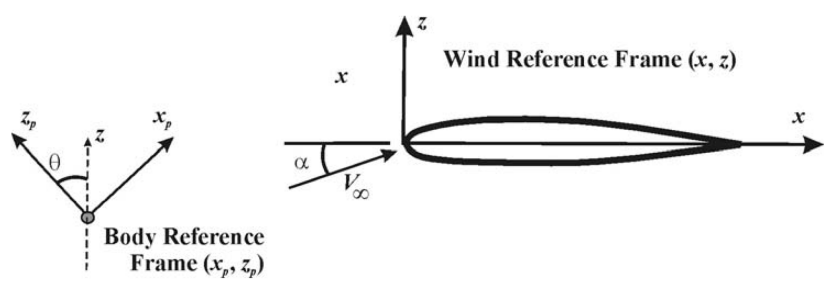

Fig. 4 The body (particle) and wind (airfoil) coordinate reference frames. relates the two reference frames to each other:

$$
\left[\begin{array}{l}
x \\
z
\end{array}\right]=\left[\begin{array}{cc}
\cos \theta & -\sin \theta \\
\sin \theta & \cos \theta
\end{array}\right]\left[\begin{array}{l}
x_{p} \\
z_{p}
\end{array}\right]
$$

where $\theta$ is the angle between the $z_{p}$ axis and the $z$ axis.

\section{Particle Trajectory Analysis}

To be able to determine the particle impingement characteristics, the trajectory of each sand particle impinging on the surface of a body needs to be determined. To accomplish this step, first, a force/ momentum balance must be applied on a particle moving through air and, second, the resulting momentum equation must be integrated with some known initial conditions until the particle impacts a surface panel or travels past the entire multi-element configuration without an impact. Similar studies related to sand and water-droplet impingement and ice accretion on aircraft and engine inlet surfaces are available in the literature $[\underline{4}-\underline{6}, \underline{8}-\underline{17}]$. The same approach has been used in this study.

The different forces acting on a particle are based on the body reference system which differs from the wind axis system by the angles of attack as shown in Fig. 4. A transformation relation similar to that given by Eq. (1) is used in switching from body to wind reference frame and vice versa. The vectors $\boldsymbol{r}_{p}$ and $\boldsymbol{V}_{p}$ represent particle position and velocity with respect to the body reference frame. The motion of the particle is governed by the particle momentum equation that can be written as

$$
m_{p} \frac{d^{2} \boldsymbol{r}_{p}}{\mathrm{~d} t^{2}}=\boldsymbol{F}_{a}+\boldsymbol{F}_{g}
$$

where $m_{p}$ is the particle mass, $\boldsymbol{F}_{a}$ the aerodynamic force (pressure and shear), and $\boldsymbol{F}_{g}$ the gravity force. The gravity force is related to the weight of the particle as follows:

$$
\boldsymbol{F}_{g}=m_{p} g(\sin \theta \boldsymbol{i}-\cos \theta \boldsymbol{k})=\rho_{p} V_{p} g(\sin \theta \boldsymbol{i}-\cos \theta \boldsymbol{k})
$$

where $g$ is the gravitational acceleration. The aerodynamic force is due to the pressure and shear forces acting on the particle surface. If we consider $S_{p}$ as the particle surface, $\boldsymbol{n}$ as the normal vector on the particle surface, and $\boldsymbol{k}_{p}$ the direction of the local vertical axis, the aerodynamic force can be expressed by the relation:

$$
\boldsymbol{F}_{a}=-\int_{S_{p}}\left(p-\rho_{a} g z\right) \boldsymbol{n} \mathrm{d} S+\int_{S_{p}} \overline{\bar{\tau}} \cdot \boldsymbol{n} \mathrm{d} S
$$

The term relating the gravity force is rewritten as

$$
\begin{aligned}
& \rho_{a} \int_{S_{p}} g z \boldsymbol{n} \mathrm{d} S=\rho_{a} \int_{V_{p}} \nabla(g z) \mathrm{d} V=\rho_{a} g V_{p} \boldsymbol{k}_{p} \\
& \quad=\rho_{a} g V_{p}(-\sin \theta \boldsymbol{i}+\cos \theta \boldsymbol{k})
\end{aligned}
$$

where $V_{p}$ is the particle volume. The others terms of Eq. (4) can be written in two parts. The first, in the same direction as the velocity $\vec{U}$ (which is the flow velocity in the body reference frame), is the drag, while the second term, in the direction perpendicular to $\vec{U}$, is the lift. Here $\rho_{a}$ is the density of air, and $p$ the ambient pressure. Studies indicate that there is no lift if the particle does not have a rotational movement and keeps an axisymmetric shape along the $\vec{U}$ direction, and if the flow is irrotational. On the basis of this assumption, the lift force can be treated as zero and thus only the drag force needs to be considered. Moreover, because the small size of the particles is in the range where shear forces cannot be neglected the drag force evaluation needs to consider both pressure and shear forces. Because, such a calculation can be very demanding, a more convenient and commonly used method is to use some form of empirical correlation for the drag coefficient of the particle. Figure 5 shows a comparison of sphere drag coefficient empirical correlations proposed by various authors [18-26]. As evident from the figure, all of the correlations agree up to a $R e=1000$. In the sphere drag measurement 


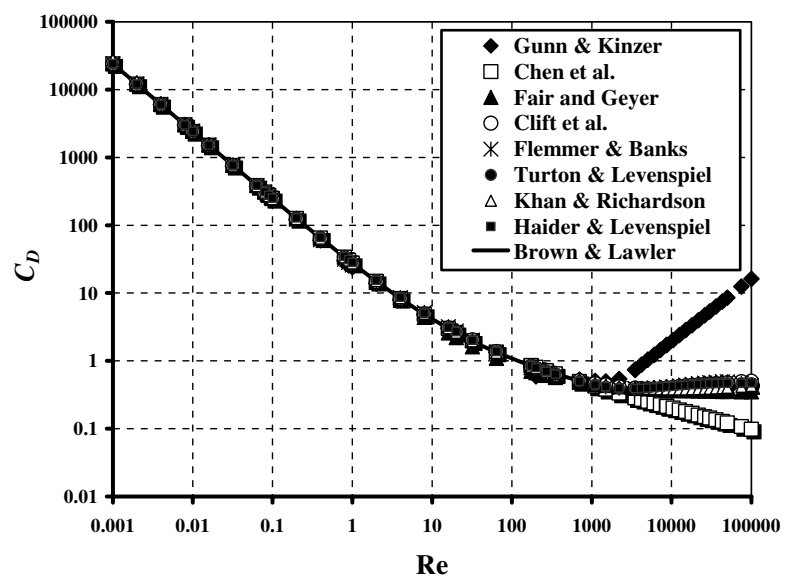

Fig. 5 Comparison of sphere drag correlations by various authors.

experiments, the terminal velocity of falling spheres in stagnant medium (air or fluid) was measured. For this terminal velocity, the drag force is equal to the weight force less the buoyancy force. With this data, it is easy to determine the drag coefficient $C_{d}$ for various Reynolds number and then, the drag force with the following relation:

$$
D=\frac{1}{2} \rho_{a} U^{2} S C_{d}
$$

In the above equation, the area $S$, which is the surface area of the particle projection on the $\vec{U}$ perpendicular plan, is unknown. To remedy this, we introduce the equivolumetric diameter $D_{\text {eq }}$ which is the diameter of the sphere with the same volume as that of the particle. Brown and Lawler [26] recently reevaluated the experimental sphere drag data available in literature to account for the effect of the walls because much of the data was measured in small diameter cylindrical vessels. They proposed new correlations for the drag coefficient $C_{d}$ based on corrected experimental data. In this study, the new sphere drag coefficient correlation based on Eq. (19) of [26] has been used because it provides the best fit to the existing experimental data for the entire range of Reynolds number $\left(10^{-3} \leq R e \leq 3.5 \times 10^{5}\right)$ considered. The correlation is given by the relation:

$$
C_{d}=\frac{24}{R e}\left(1+0.150 R e^{0.681}\right)+\frac{0.407}{1+(8710 / R e)}
$$

Because sand particles are nonspherical, drag coefficient correlations for nonspherical particles were also compared for error and range of applicability. Chhabra et al. [27] critically evaluated the widely used drag correlations from 19 studies with a resulting database of 1900 data points for a range of Reynolds number $\left(10^{-4} \leq R e \leq 5 \times 10^{5}\right)$. One of the methods investigated by Chhabra et al. was the Haider and Levenspiel's nonspherical correlation [25]. The Haider and Levenspiel correlation is valid for the particle Reynolds number less than $2.5 \times 10^{5}$. The maximum particle Reynolds number observed in this study (for very fine to coarse size particles and a velocity of $40 \mathrm{~m} / \mathrm{s}$ ) was of the order of $100-1500$, respectively. Haider and Levenspiel relate the shape of a nonspherical particle by a shape factor $\phi$ which is defined as the ratio of the surface area of a sphere having the same volume as the particle to the actual surface area of the particle. Thus for nonspherical particles, $0<\phi<1$. The sand particle shape factor can vary from 0.3 to as high as 0.9 . The drag coefficient correlation of Haider and Levenspiel for nonspherical particles with a shape factor $\phi$ is given by

$$
C_{d}=\frac{24}{R e}\left(1+b_{1} R e^{b_{2}}\right)+\frac{b_{3}+R e}{b_{4}+R e}
$$

where

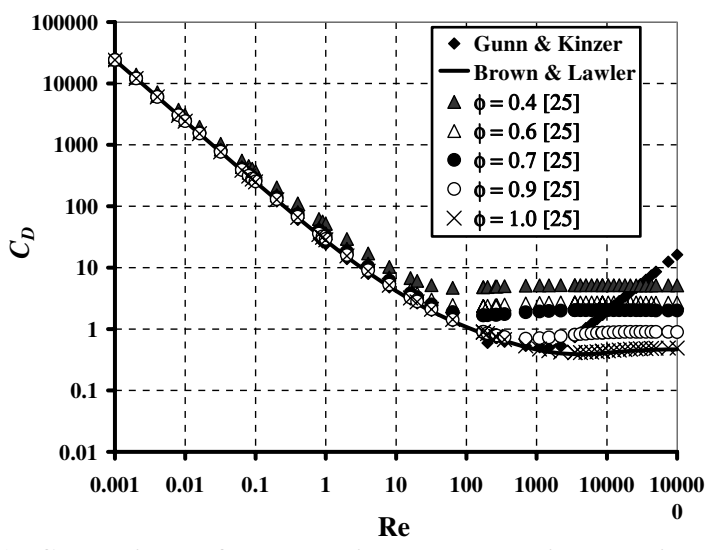

Fig. 6 Comparison of nonspherical and spherical particle drag correlations by various authors.

$$
\begin{aligned}
& b_{1}=\exp \left(2.3288-6.4581 \phi+2.4486 \phi^{2}\right) \\
& b_{2}=0.0964+1.5565 \phi \\
& b_{3}=\exp \left(4.905-13.8944 \phi+18.4222 \phi^{2}-10.2599 \phi^{3}\right) \\
& b_{4}=\exp \left(1.4681+12.258 \phi-20.7322 \phi^{2}+15.8855 \phi^{3}\right)
\end{aligned}
$$

The results of Chhabra et al. [27] indicate that Haider and Levenspiel's correlation satisfactorily predicts drag for particles with values of $\phi>0.67$. Figure 6 shows a comparison of the Haider and Levenspiel correlation (for nonspherical particles) prediction with other methods. It is evident from the figure that for small values of shape factor (elongated shape), a significant increase in drag results. Thus, the shape factor (or the sphericity) of the sand particle should be given due consideration in the design of such an IPS system. In the current study, the shape factor of the sand particle has been assumed as 1. And the drag coefficient correlation of Brown and Lawler, Eq. (7), is considered.

Finally, the aerodynamic force is given by the following relation:

$$
\boldsymbol{F}_{a}=\rho_{a} g V_{p}(-\sin \theta \boldsymbol{i}+\cos \theta \boldsymbol{k})+\frac{1}{2} \rho_{a} S C_{d} U \boldsymbol{U}
$$

Substituting the above expressions for both aerodynamic force and gravity force in the particle momentum equation, Eq. (2), yields

$$
\rho_{p} V_{p} \frac{\mathrm{d}^{2} \boldsymbol{r}_{p}}{\mathrm{~d} t^{2}}=\left(\rho_{a}-\rho_{p}\right) g V_{p}(-\sin \theta \boldsymbol{i}+\cos \theta \boldsymbol{k})+\frac{1}{2} \rho_{a} S C_{d} U \boldsymbol{U}
$$

By assuming that the particle surface area and volume are $S=$ $\pi D_{\mathrm{eq}}^{2} / 4$ and $V_{p}=\pi D_{\mathrm{eq}}^{3} / 6$, respectively, and that the Reynolds number based on equivolumetric particle diameter $D_{\text {eq }}$ is $R e=\rho_{a} D_{\text {eq }} U / \mu_{a}$, the previous equation can be rewritten as

$$
\frac{\mathrm{d}^{2} \boldsymbol{r}_{p}}{\mathrm{~d} t^{2}}=\frac{\rho_{a}-\rho_{p}}{\rho_{p}} g(-\sin \theta \boldsymbol{i}+\cos \theta \boldsymbol{k})+\frac{3 C_{d} R e \mu}{4 \rho_{p} D_{\mathrm{eq}}^{2}} \boldsymbol{U}
$$

Finally, introducing two parameters $K_{g}=\left(\rho_{p}-\rho_{a}\right) g / \rho_{p}$ and $K_{a}=\rho_{p} D_{\text {eq }}^{2} /\left(18 \mu_{a}\right)$ in the above equation and noting that $\boldsymbol{U}=\boldsymbol{V}_{a}-\boldsymbol{V}_{p}$, yields

$$
\frac{\mathrm{d}^{2} \boldsymbol{r}_{p}}{\mathrm{~d} t^{2}}+\frac{C_{d} R e}{24 K_{a}} \frac{\mathrm{d} \boldsymbol{r}_{p}}{\mathrm{~d} t}=K_{g}(\sin \theta \boldsymbol{i}-\cos \theta \boldsymbol{k})+\frac{C_{d} R e}{24 K_{a}} \boldsymbol{V}_{a}
$$

where $\boldsymbol{V}_{a}=u_{a} \boldsymbol{i}+w_{a} \boldsymbol{k}$. Note that this second-order differential equation is nonlinear because of the term $C_{d} R e / 24 K_{a}$, which depends on the particle position and the velocity. The difficulty to determine the term $C_{d} R e / 24 K_{a}$ suggests that a numerical technique must be employed to integrate the momentum equation (12). 


\section{Momentum Equation Integration}

The momentum equation (12) can also be written as

$$
\frac{\mathrm{d}^{2} \boldsymbol{r}_{p}}{\mathrm{~d} t^{2}}+\delta \frac{\mathrm{d} \boldsymbol{r}_{p}}{\mathrm{~d} t}=\boldsymbol{\beta}
$$

where

$$
\boldsymbol{\beta}=\left(\delta u_{a}+K_{g} \sin \theta\right) \boldsymbol{i}+\left(-K_{g} \cos \theta+\delta w_{a}\right) \boldsymbol{k}
$$

and

$$
\delta\left(\boldsymbol{r}_{p}, \boldsymbol{V}_{p}\right)=\frac{C_{d} R e}{24 K_{a}}
$$

The above momentum equation, which is a second-order differential equation, can be decomposed into two first-order differential equations:

$$
\frac{\mathrm{d} \boldsymbol{V}_{p}}{\mathrm{~d} t}=\boldsymbol{\beta}-\delta \boldsymbol{V}_{p}=\boldsymbol{f}\left(\boldsymbol{r}_{p}, \boldsymbol{V}_{p}\right) \quad \text { and } \quad \frac{\mathrm{d} \boldsymbol{r}_{p}}{\mathrm{~d} t}=\boldsymbol{V}_{p}
$$

The well-known fourth-order Runge-Kutta method [28] is used to integrate the above nonlinear equations. Starting with the current particle position $\boldsymbol{r}_{p, i}=\left(x_{p, i}, z_{p, i}\right)$ and velocity $\boldsymbol{V}_{p, i}=\left(u_{p, i}, w_{p, i}\right)$, the new postions $\boldsymbol{r}_{p, i+1}=\left(x_{p, i+1}, z_{p, i+1}\right)$ and velocity $\boldsymbol{V}_{p, i+1}=$ $\left(u_{p, i+1}, w_{p, i+1}\right)$ are calculated with the aid of the following relations based on the Runge-Kutta method [28]:

$$
\begin{aligned}
& \boldsymbol{r}_{p, i+1}=\boldsymbol{r}_{p, i}+\tau \boldsymbol{V}_{p, i}+\frac{\tau}{6}\left(\boldsymbol{k}_{1}+\boldsymbol{k}_{2}+\boldsymbol{k}_{3}\right) \\
& \boldsymbol{V}_{p, i+1}=\boldsymbol{V}_{p, i}+\frac{1}{6}\left(\boldsymbol{k}_{1}+2 \boldsymbol{k}_{2}+2 \boldsymbol{k}_{3}+\boldsymbol{k}_{4}\right)
\end{aligned}
$$

The four coefficients $\boldsymbol{k}_{1}, \boldsymbol{k}_{2}, \boldsymbol{k}_{3}$, and $\boldsymbol{k}_{4}$ in the above equation are given by

$$
\begin{gathered}
\boldsymbol{k}_{1}=\tau \boldsymbol{f}\left(\boldsymbol{r}_{p, i}, \boldsymbol{V}_{p, i}\right) \\
\boldsymbol{k}_{2}=\tau \boldsymbol{f}\left(\boldsymbol{r}_{p, i}+\frac{\tau}{2} \boldsymbol{V}_{p, i}, \boldsymbol{V}_{p, i}+\frac{1}{2} \boldsymbol{k}_{1}\right) \\
\boldsymbol{k}_{3}=\tau \boldsymbol{f}\left(\boldsymbol{r}_{p, i}+\frac{\tau}{2} \boldsymbol{V}_{p, i}+\frac{\tau}{4} \boldsymbol{k}_{1}, \boldsymbol{V}_{p, i}+\frac{1}{2} \boldsymbol{k}_{2}\right) \\
\boldsymbol{k}_{4}=\tau \boldsymbol{f}\left(\boldsymbol{r}_{p, i}+\boldsymbol{V}_{p, i}+\frac{\tau}{2} \boldsymbol{k}_{2}, \boldsymbol{V}_{p, i}+\boldsymbol{k}_{3}\right)
\end{gathered}
$$

where $\tau$ is the integration time step. This time step must neither be too small to result in a long computation time nor too large that it leads to inaccuracies in computation. A set of initial conditions are required to start the integration. These initial conditions are taken at an upstream point in space where the flow is unperturbed; that is, the flow velocity at this point must not differ from the freestream $\boldsymbol{V}_{\infty}$ value by more than $1 \%$. The following relation gives the initial velocity of the particle:

$$
\boldsymbol{V}_{p}^{0}=\boldsymbol{V}_{a}^{0}-\boldsymbol{U}^{0}
$$

with $\boldsymbol{V}_{a}^{0}$ the flow velocity in the unperturbed flow, that is $\boldsymbol{V}_{\infty}$, and $\boldsymbol{U}^{0}$ the terminal velocity of the falling particle. The terminal velocity $\boldsymbol{U}^{0}$ can be calculated with the relation

$$
\frac{C_{d} R e}{24} U^{0}=K_{a} K_{g}
$$

If we consider particles with an equivolumetric diameter between 10 and $80 \mu \mathrm{m}$, the Stokes law can be applied and the previous equation becomes $U^{0}=K_{a} K_{g}$. Consequently, the initial particle velocity is

$$
u_{p}^{0}=u_{\infty} \quad \text { and } \quad w_{p}^{0}=w_{\infty}-K_{a} K_{g}
$$

If the equivolumetric diameter is greater than $80 \mu \mathrm{m}$, the previous equation becomes inaccurate and the velocity calculated is greater than the true velocity. By using the calculated velocity in the momentum equation, acceleration results that ultimately leads to the particle terminal velocity.

\section{Impingement Location Determination}

The particle trajectories are initiated at a distance of about five chord lengths (of the middle airfoil representing the engine centerline) and are calculated until they either impact any of the airfoil elements or go around them. The location of the particle impingement point on any airfoil element surface is determined using a systematic search approach. While the particle is upstream of any airfoil element, no impact or impingement search is performed. Once the particle reaches the border or the bounding box around any of the elements along the $x$ axis, a search is initiated that checks for any impingement on surface panels of all elements with the knowledge of the particle position $(x, z)$. This is accomplished as follows.

First of all, the particle trajectory is assumed to be a straight line, from the old position $\left(x_{i}, z_{i}\right)$ to the new position $\left(x_{i+1}, z_{i+1}\right)$. Each airfoil element surface is represented by means of a number of flat and straight panels. To determine whether an impact has occurred on a panel [represented by the vertices $\left(x_{1}, z_{1}\right)$ and $\left(x_{2}, z_{2}\right)$ ] or not, the following two conditions are verified:

$$
x_{\min } \leq x_{i+1} \quad \text { and } \quad x_{i} \leq x_{\max }, \quad z_{\min } \leq z_{i+1} \quad \text { and } \quad z_{i} \leq z_{\max }
$$

with

$$
\begin{aligned}
& x_{\min }=\min \left(x_{1}, x_{2}\right) \quad \text { and } \quad z_{\min }=\min \left(z_{1}, z_{2}\right) \\
& x_{\max }=\max \left(x_{1}, x_{2}\right) \quad \text { and } \quad z_{\max }=\max \left(z_{1}, z_{2}\right)
\end{aligned}
$$

When all of the above conditions are satisfied for a panel, the impact takes place on that panel. The location of the impact point is calculated by considering the particle trajectory parametric equations given by

$$
\begin{aligned}
& x_{i+1}=x_{i}+t_{0}\left(x_{i+1}-x_{i}\right)=x_{i}+t_{0} l_{0} \\
& z_{i+1}=z_{i}+t_{0}\left(z_{i+1}-z_{i}\right)=z_{i}+t_{0} n_{0}
\end{aligned}
$$

where $\left(l_{0}, n_{0}\right)$ is the trajectory direction vector and $t_{0}$ is the parameter related to that trajectory. All points that belong to the trajectory corresponding to the impingement or impact must satisfy $0 \leq t_{0} \leq 1$. Similarly, the parametric equations for each panel are

$$
\begin{aligned}
& x=x_{2}+t_{1}\left(x_{1}-x_{2}\right)=x_{2}+t_{1} l_{1} \\
& z=z_{2}+t_{1}\left(z_{1}-z_{2}\right)=z_{3}+t_{1} n_{1}
\end{aligned}
$$

And all points located on the panel must satisfy the following additional condition:

$$
0 \leq t_{1} \leq 1
$$

Hence, the two parameters $t_{0}$ and $t_{1}$ determine whether the particle trajectory intercepts a surface panel or not. Numerically, $t_{0}$ is calculated first and checked to see if it satisfies the condition $0 \leq t_{0} \leq 1$. If this condition is satisfied, only then is $t_{1}$ calculated to verify whether there is any impact on the panel. Finally, the coordinates of the impact point are found from $t_{0}$ and $\left(l_{0}, n_{0}\right)$.

This procedure is repeated for each particle trajectory. The particle trajectories are initiated by releasing the particles from different upstream locations $\left(z_{0}\right)$ along the $z$ axis (Fig. 7) while keeping a constant upstream distance of five chord lengths $\left(x_{0}=-5 c\right)$. Thus, the impingement regions on individual elements are determined by an appropriate sweep of the $z$ axis. Because these impingement 


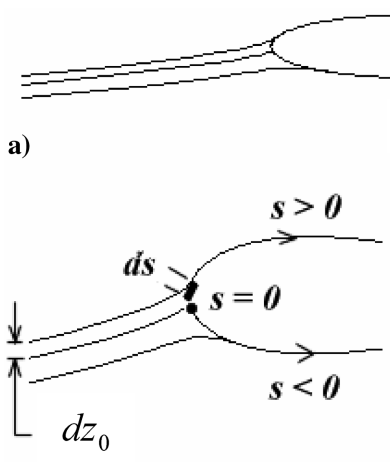

b)

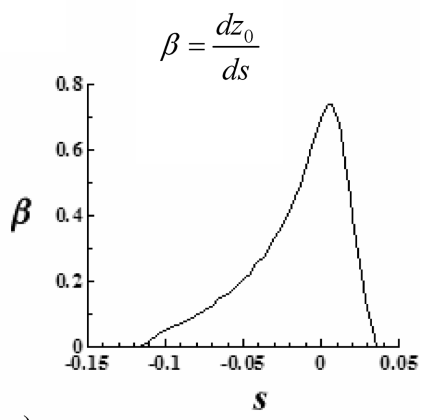

c)

Fig. 7 a) Particle trajectories; b) close-up view and nomenclature; and c) a typical local impingement efficiency $\beta$ curve [10].

regions depend upon the aerodynamic characteristics of the multielement airfoil configuration, in terms of local flow circulation, and the particle characteristics, a tradeoff study becomes imperative to gain insight into the design of such systems.

\section{Impingement Characteristics Analysis}

The impingement characteristics of an airfoil element [10] are defined by the impingement efficiency $\beta$, which is the dimensionless mass flux of material impinging at a particular point on the airfoil surface. It is nondimensionalized with respect to the mass flux in the freestream. The term impingement efficiency appears very frequently in studies related to aircraft ice accretion and waterdroplet impingement where it serves to quantify the amount of ice that may accrete on an aircraft surface and hence an important consideration in the design of an aircraft anti-icing system. In this study, the idea of impingement efficiency has been taken from an aircraft icing field to validate and establish the accuracy of the method. Hence, to establish the validity of the method, the particle is treated as a water droplet.

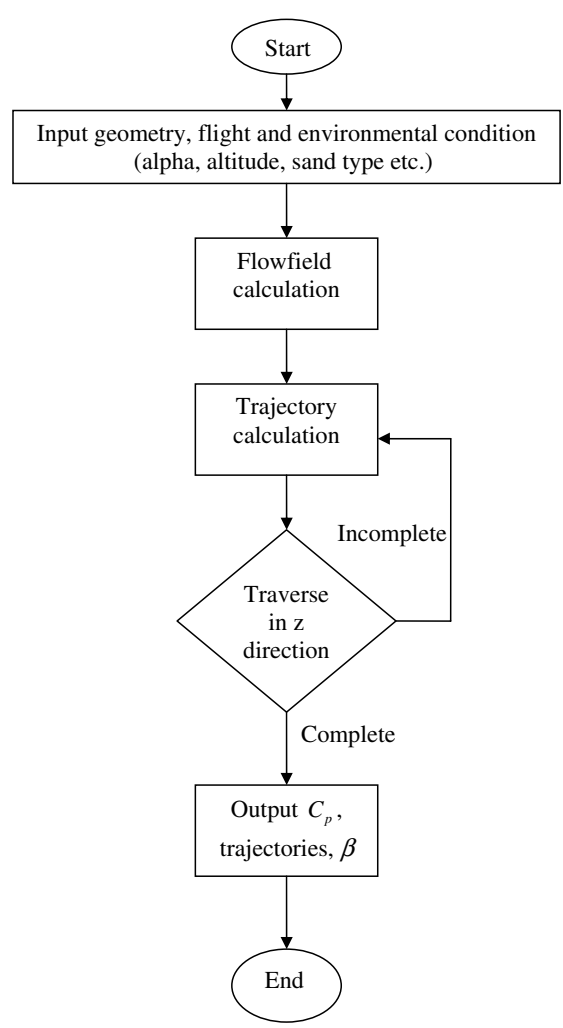

Fig. 8 Flowchart of numerical procedure.

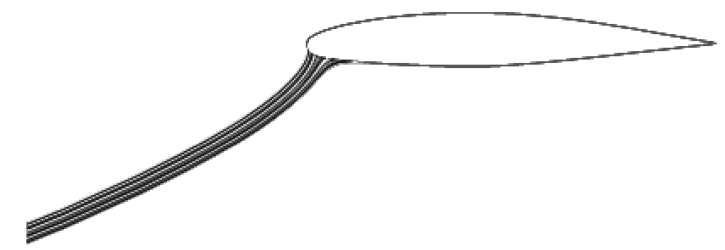

Fig. 9 Particle trajectories around a single airfoil.

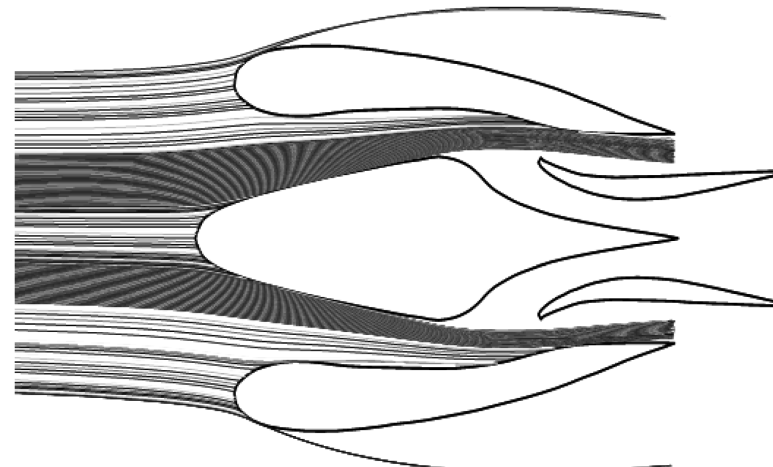

Fig. 10 Particle trajectories around a five-element airfoil configuration simulating an IPS system.

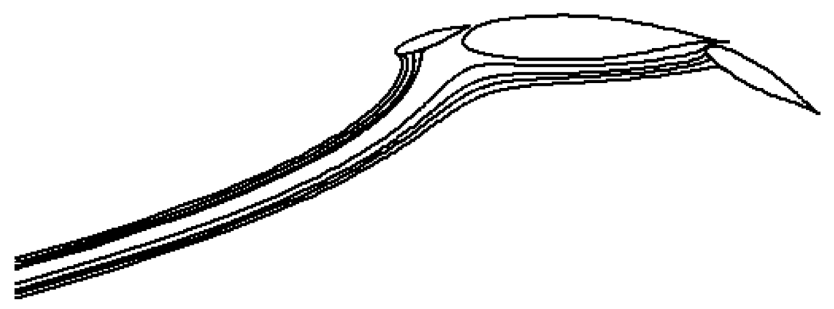

a)

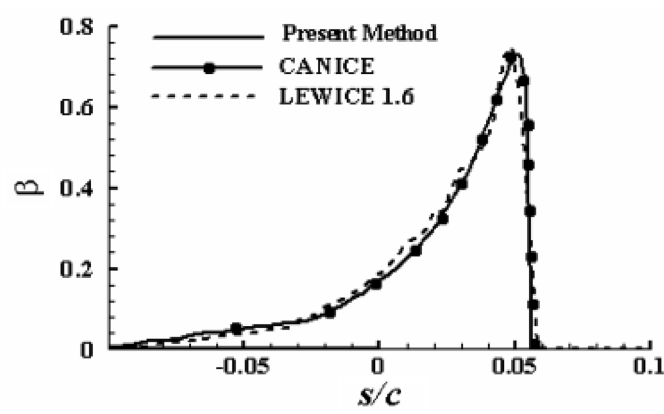

b)

Fig. 11 Comparison of a) water-droplet trajectories for a threeelement airfoil configuration, and b) impingement efficiencies of the slat predicted by three different methods.

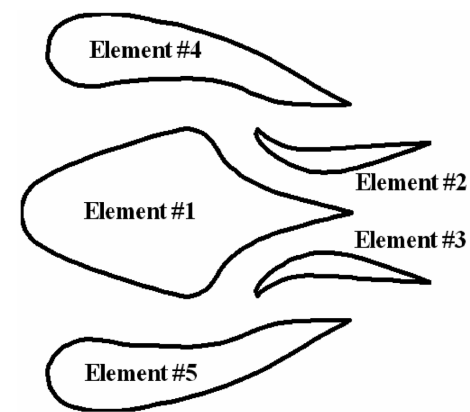

Fig. 12 A five-element airfoil configuration model of an IPS system. 


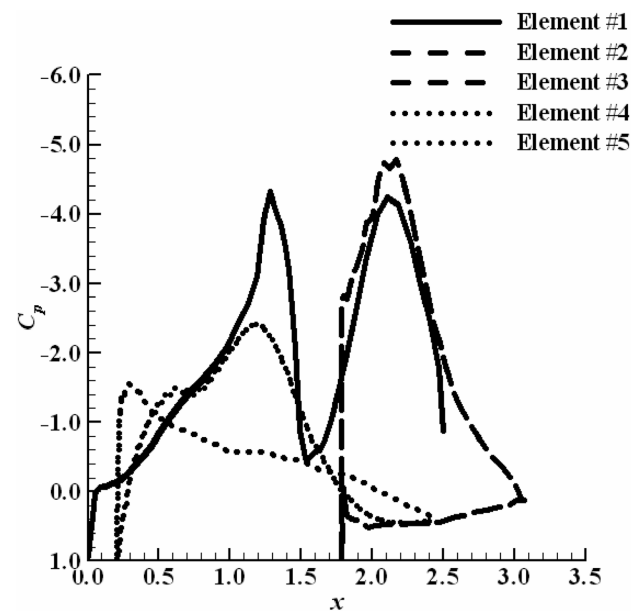

Fig. 13 Surface $C_{p}$ distribution on all the elements.

Therefore, in the case of water droplets, the mass of water between two consecutive trajectories a distance $\delta z_{0}$ apart must then be deposited over a corresponding region $\delta s$ of the airfoil surface. Then, in the limit, the local impingement efficiency $\beta$ can be defined as

$$
\beta=\frac{\mathrm{d} z_{0}}{\mathrm{~d} s}
$$

where $s$ is the surface arc length measured from the airfoil element leading edge. Figure 7 from [10] illustrates the nomenclature, the concept behind the impingement efficiency as well as a typical impingement efficiency curve for an airfoil at a positive angle of attack. The impingement efficiency curve indicates that for positive angle of attack, more impingement occurs on the lower surface $(s<0)$ as compared to the upper surface $(s>0)$. The maximum value of the impingement efficiency appears at the stagnation point.

\section{Numerical Implimentation}

The flowfield, trajectory, and impingement characteristics calculation modules were implemented in a FORTRAN code. Figure $\underline{8}$ shows the flowchart of the numerical method. Figure 9 shows sand particle trajectories impinging on a single airfoil while Fig. 10 shows sand particle trajectories around an IPS system modeled as a five-element airfoil configuration.

\section{Validation Example}

A three-element (slat, main, and flap) airfoil configuration shown in Fig. 11a was used for validation of the method. The airfoil configuration is flying at an altitude of $580.6 \mathrm{~m}$, with an angle of attack $\alpha=6 \mathrm{deg}$, a velocity of $90 \mathrm{~m} / \mathrm{s}$, an ambient temperature of $-10^{\circ} \mathrm{C}$, and a Reynolds number, based on chord length, of $6.52 \times 10^{6}$. The liquid water content (LWC) in the cloud is $0.54 \mathrm{~g} / \mathrm{m}^{3}$ and the mean volumetric diameter $\left(D_{\text {eq }}\right)$ is $20 \mu \mathrm{m}$. Figure 11a shows the water-droplet trajectories for the three-element airfoil configuration predicted by the current method. For this validation example, the sphere drag correlation of Gunn and Kinzer [18] was used. A comparison of the impingement efficiencies of the slat element predicted by the present and two state-of-the-art droplet impingement and ice accretion simulation methods, CANICE $[\underline{8}, \underline{10}, \underline{15}-17]$ and LEWICE [9], as shown in Fig. $11 \mathrm{~b}$, indicates that the results predicted by the present method are accurate and reliable.

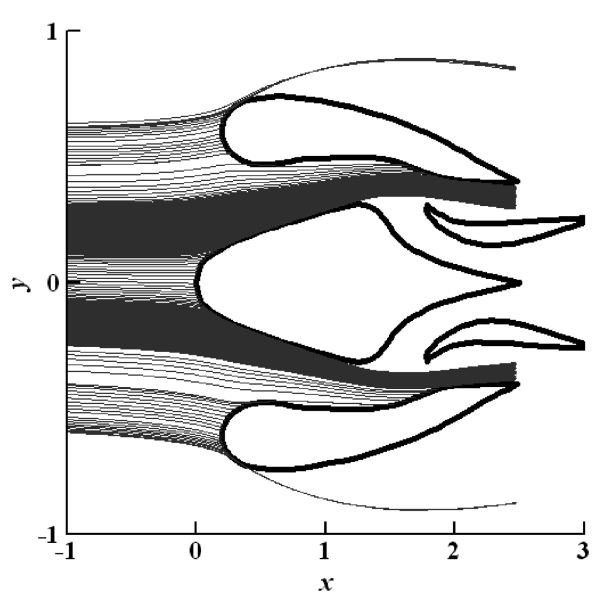

a)

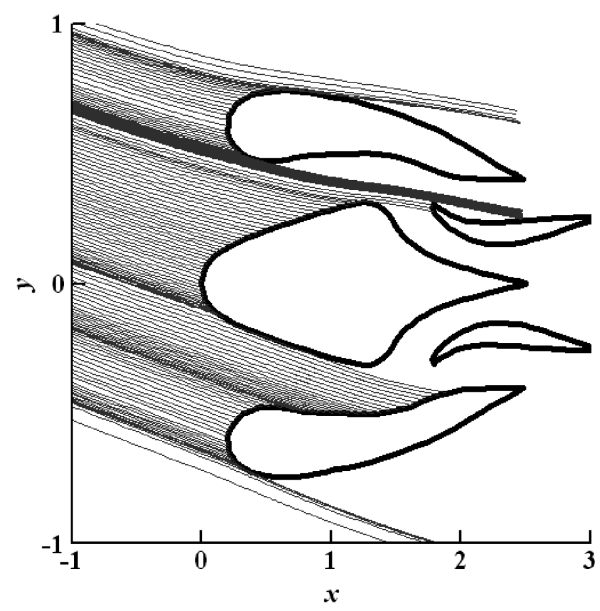

c)

Fig. 14 Plot of sand particle trajectories of a) very fine, b) fine, c) medium, and d) coarse sand particles.

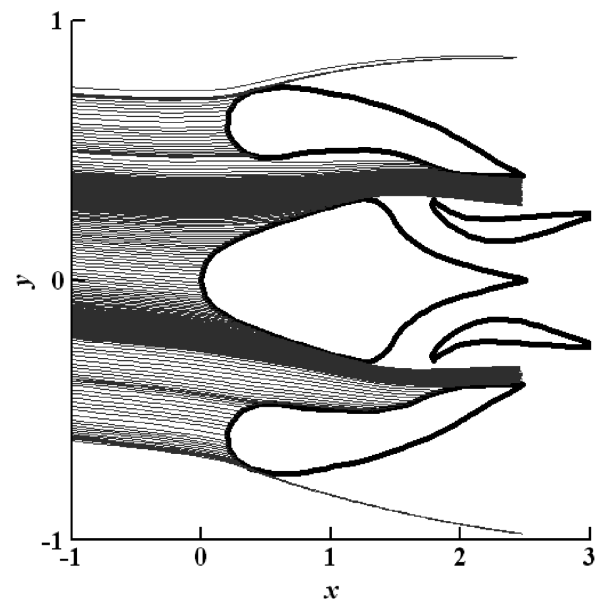

b)

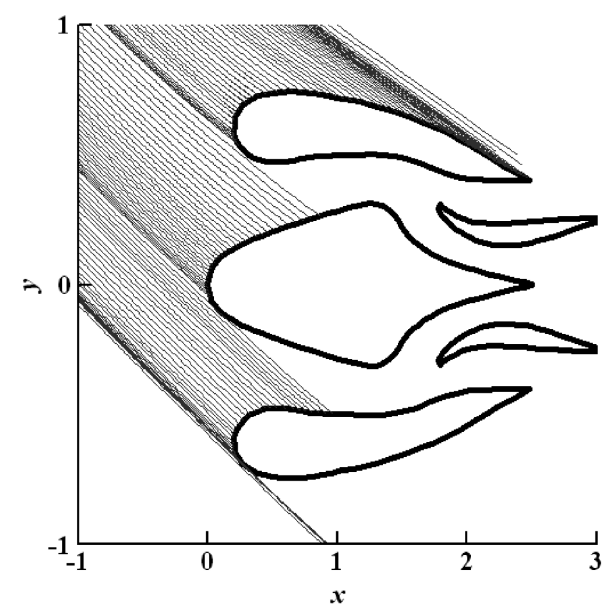

d) 


\section{Numerical Results and Discussion}

The results that follow have been obtained for an IPS system modeled using five-element airfoil configuration as shown in Fig. 12. In these cases, the angle of attack has been fixed at $0 \mathrm{deg}$, whereas the freestream velocity is kept at $40 \mathrm{~m} / \mathrm{s}$. Figure 13 shows the pressure coefficient distribution on the five airfoil elements of the IPS system model predicted by the flow analysis module.

Figure 14 shows the effect of different types of sand particles, in terms of different particle diameters, on impingement trajectories. The influence of gravity on the particle trajectory becomes more and more apparent and significant as the particle size is increased from very fine to coarse. Figures $14 \mathrm{c}$ and $14 \mathrm{~d}$ reveal that for medium to coarse sand particles, the flow circulation strength in this particular example has very little influence on the particle trajectories and that the particle trajectories remain almost unaltered due to inertia for this particular configuration. This limitation can be overcome by a detailed study into the design of such configurations. Another limitation of the current analysis method is also apparent from Fig. 14 in that the sand particle trajectories are terminated after impingement on the surface. In reality, the sand particles bounce back from the impact and reenter the air stream. On impact, the particles lose part of their energy and hence the reflected velocity of the particle is lower than the incoming velocity. This impact is described by the momentum-based coefficient of restitution which is greatly dependent upon the impact angle. To accurately predict the particle trajectories an appropriate rebound model, experiment based [29] or statistical [30-32], describing the particle-surface collision must be used in the impingement analysis module.

Figure 15 shows the effect of the different types of sand particles on impingement characteristics in terms of the influx of sand particles and the impact locations on the surface of the different elements. In this figure, the surface arc length $s$ is normalized by the chord length $c$ of the middle airfoil element. The figure indicates the maximum impingement location and the limits or the extent of impingement on the surface of the different elements of the IPS system model. For medium to coarse sand particles, the impingement regions are limited to the upper surfaces of the elements only as shown in Figs. $15 \mathrm{c}$ and $15 \mathrm{~d}$. Maximum impingement efficiency $\beta$ for a particular element is found to be in the vicinity of the element leading edge. The limits of impingement or impingement regions together with given flight and environmental conditions can be used in parametric studies to provide an insight into the design of such IPS systems.

\section{IPS System Design Methodology}

The results of impingement analysis suggest that the design of an IPS system model based on a five-element airfoil configuration can easily be preformed in a direct design approach in which design is achieved through analysis. The design objective in this case could be as follows:

1) To ensure the required air mass flow rate for the engine through elements 2 and 3 (see Fig. 12). This can be translated into an optimum inlet area or a minimum distance between elements 2 and 3 .

2) To ensure that no sand particle trajectory enters the engine in between elements 2 and 3 .

With these objectives in mind, the impingement analysis can be performed for different operating conditions for the engine as well as environmental conditions (types of sand or dust in air) to find the appropriate position of elements 2 and 3 or even 1 . This can be achieved by coupling the impingement analysis with an optimization program along with the above objective and constraints. This direct design approach may, however, be costly both in terms of resources as well as time.

A more better and thorough design could be achieved by actually designing the sectional profile of the different airfoil elements (Fig. 12) in the above model to satisfy the constraints listed above in addition to the closure and far-field constraints on the airfoil geometry using the inverse airfoil design method $[\underline{33}, \underline{34}]$ with

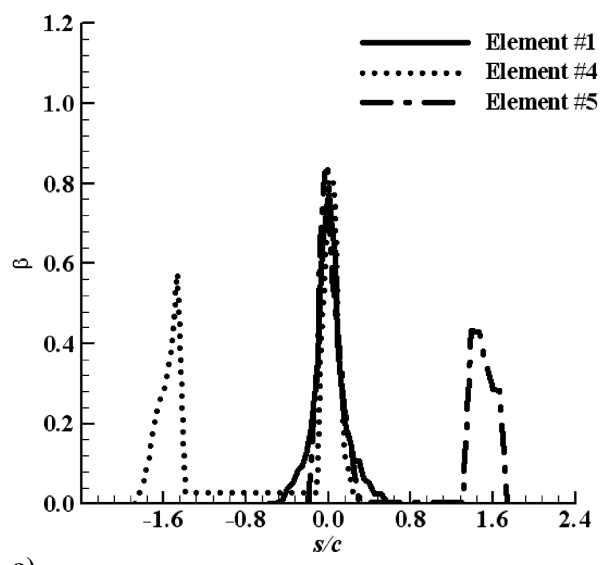

a)

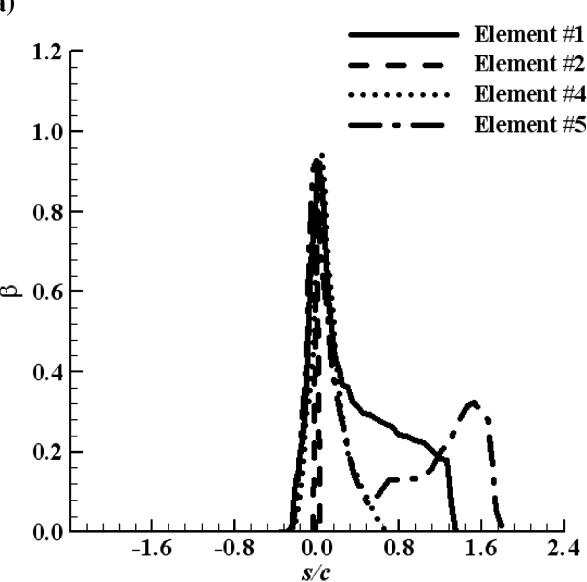

c)

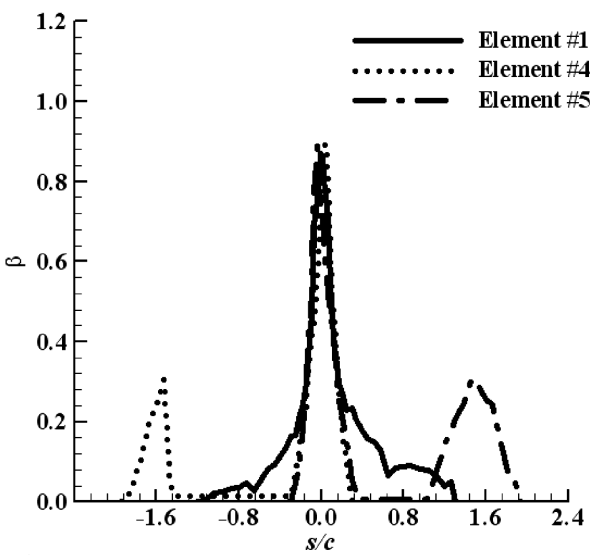

b)

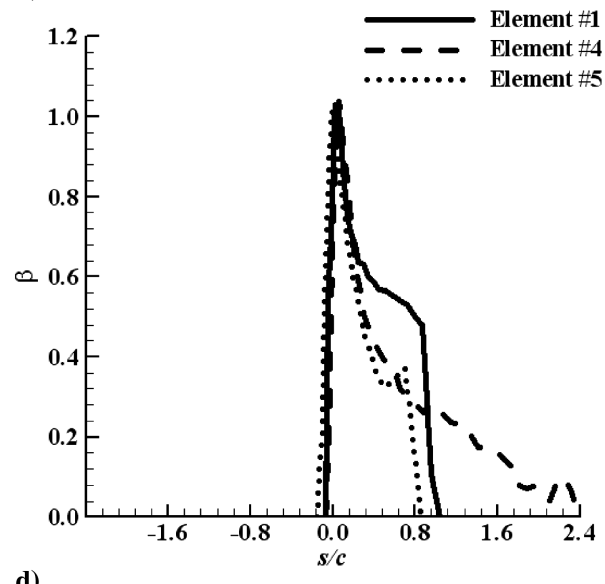

Fig. 15 Plot of sand particle impingement characteristics of a) very fine, b) fine, c) medium, and d) coarse sand particles. 
multivariable and multipoint design capability. Such a multivariable and multipoint inverse design tool could be used interactively to perform rapid trade studies to examine the potential payoff for a proposed IPS system. Furthermore, tradeoffs could be performed and alternatives could be explored to gain insight into a more thorough design of such multi-element airfoil based IPS systems. Hence the analysis tool developed here could serve as a very important and useful tool in exploring the design space and developing a more efficient design methodology.

\section{Conclusions}

In this paper, a numerical tool for the flowfield, trajectory, and impingement analyses of a multi-element airfoil based IPS system has been developed. The usefulness of the tool is its use in the design of such a multi-element airfoil based model of an IPS systems in that it can be coupled to a multi-element and multipoint airfoil design tool. With such a design and analysis tool at hand, the design space can be explored as well as tradeoff studies can be performed that can aid in the development of design methodology for multi-element airfoil based IPS systems. The analysis tool is able to perform impingement analysis using spherical, nonspherical solid particles as well as water droplets for a range of Reynolds number $\left(10^{-4} \leq R e \leq 5 \times 10^{5}\right)$. A limitation of the analysis tool is that it lacks an appropriate particle rebound model for the treatment of particle-wall collisions.

Two different design methodologies for the design of the multielement airfoil based IPS system have been proposed that can make use of the analysis program developed in this study. These design methodologies require coupling of the impingement analysis program to an optimization tool or a multivariable and multipoint inverse airfoil design tool.

\section{Acknowledgement}

The authors are grateful to King Fahd University of Petroleum and Minerals, Dhahran, Saudi Arabia, for its support in accomplishing this study.

\section{References}

[1] Tabakoff, W., and Hamed, A., "Installed Engine Performance in Dust Laden Atmosphere," AIAA Paper 1984-2488, 1984.

[2] van der Walt, J. P., and Nurick, A., "Prediction of Helicopter Engines Fitted with Dust Filters," Journal of Aircraft, Vol. 23, No. 1, Jan.Feb. 1995, pp. 118-123.

[3] Mann, D., "Helicopter Engine Particle Separator," www.triz-journal. com/archives/1999/02/a/index.htm, University of Bath, 1998

[4] Vittal, B. V. R., Tipton, D. L., and Bennett, W. A., "Development of an Advanced Vaneless Inlet Particle Separator for Helicopter Engines," Journal of Propulsion and Power, Vol. 2, No. 5, 1986, pp. 438-444.

[5] Breitman, D. S., Dueck, E. G., and Habashi, W. G., "Analysis of a SplitFlow Inertial Particle Separator by Finite Elements," Journal of Aircraft, Vol. 22, No. 2, 1985, pp. 135-140.

[6] Zedan, M., Mostafa, A., Hartman, P., and Sehra, A., "Viscous Flow Analysis of Advanced Particle Separators," Journal of Propulsion and Power, Vol. 8, No. 4, 1992, pp. 843-848.

[7] Tuncer, C., An Engineering Approach to the Calculation of Aerodynamic Flows, Horizon Publishing Inc. (Springer), CA, 1999.

[8] Saeed, F., Brette, C., Fregeau, M., Trifu, O., and Paraschivoiu, I., "A Three-Dimensional Water Droplet Trajectory and Impingement Analysis Program," AIAA Paper 2005-4838, June 2005.

[9] Ruff, G. A., and Berkowitz, B. M., "Users Manual for the NASA Lewis Ice Accretion Prediction Code (LEWICE)," NASA CR-185129, May 1990

[10] Saeed, F., "State-of-the-Art Aircraft Icing and Anti-Icing Simulation,"
ARA (American Romanian Academy) Journal, Vols. 2000-2002, Nos. 25-27, June 2002.

[11] Langmuir, I., and Blodgett, K. B., "A Mathematical Investigation of Water Droplet Trajectories," U.S. Army Air Forces TR 5418, Feb. 1946 (Contract No. W-33-038-ac-9151 with General Electric Co.); also U.S. Department of Commerce Publication Board (PB) No. 27565.

[12] Bragg, M. B., "A Similarity Analysis of the Droplet Trajectory Equation," AIAA Journal, Vol. 20, No. 12, Dec. 1982, p. 1681.

[13] Wells, S. L., and Bragg, M. B., "A Computational Method for Calculating Droplet Trajectories Including the Effects of Wind Tunnel Walls," AIAA Paper 92-0642, Jan. 1992.

[14] Bergrun, N. R., "A Method for Numerically Calculating the Area and Distribution of Water Impingement on the Leading Edge of an Airfoil in a Cloud," NACA TN 1397, Aug. 1947.

[15] Tran, P., Brahimi, M. T., Paraschivoiu, I., Pueyo, A., and Tezok, F., "Ice Accretion on Aircraft Wings with Thermodynamic Effects," Journal of Aircraft, Vol. 32, No. 2, March-April 1995, pp. 444-446.

[16] Tran, P., Brahimi, M. T., and Paraschivoiu, I., "Ice Accretion on Aircraft Wings," Canadian Aeronautics and Space Journal, Vol. 40, No. 3, Sept. 1994, pp. 91-98.

[17] Paraschivoiu, I., Tran, P., and Brahmi, M. T., "Prediction of Ice Accretion with Viscous Effects on Aircraft Wings," Journal of Aircraft, Vol. 31, No. 4, July-Aug. 1994, pp. 855-861.

[18] Gunn, R., and Kinzer, G. D., "The Terminal Velocity of the Fall of Water Droplets in Stagnant Air," Journal of Meteorology, Vol. 6, 1949, pp. 243-248.

[19] Chen, X., McLaury, B. S., and Shirazi, S. A., "Application and Experimental Validation of a Computational Fluid Dynamics (CFD)Based Erosion Prediction Model in Elbows and Plugged Tees," Computers and Fluids, Vol. 33, 2004, pp. 1251-1272.

[20] Fair, G., and Geyer, J., Water Supply and Waste Water Disposal, Wiley, New York, 1954.

[21] Clift, R., Grace, J. R., and Weber, M. E., Bubbles, Drops, and Particles, Academic Press, New York, 1978.

[22] Flemmer, R. L. C., and Banks, C. L., "On the Drag Coefficient of a Sphere," Powder Technology, Vol. 48, No. 3, 1986, pp. 217-221.

[23] Turton, R., and Levenspiel, O., "A Short Note on the Drag Correlation for Spheres," Powder Technology, Vol. 47, 1986, pp. 83-86.

[24] Khan, A. R., and Richardson, J. F., "The Resistance to Motion of a Solid Sphere in a Fluid," Chemical Engineering Communications,Vol. 62, 1987, pp. 135-150.

[25] Haider, A., and Levenspiel, O., "Drag Coefficient and Terminal Velocity of Spherical and Nonspherical Particles," Powder Technology, Vol. 58, 1989, pp. 63-70.

[26] Brown, P. P., and Lawler, D. F., "Sphere Drag and Settling Velocity Revisited," Journal of Environmental Engineering, Vol. 129, No. 3, March 2003, pp. 222-231.

[27] Chhabra, R. P., Agarwal, L., and Sinha, N. K., "Drag on Non-Spherical Particles: An Evaluation of Available Methods," Powder Technology, Vol. 101, No. 3, March 1999, pp. 288-295.

[28] Fehlberg, E., "Classical Eighth- and Lower-Order Runge-KuttaNyström Formulas with a New Stepsize Control Procedure for Special Second-Order Differential Equations," NASA TR R-381, March 1972.

[29] Hamed, A., Jun, Y. D., and Yeuan, J. J., "Particle Dynamics Simulations in Inlet Separator with an Experimentally Based Bounce Model," Journal of Propulsion and Power, Vol. 11, No. 2, MarchApril 1995, pp. 230-240.

[30] Grant, T., and Tabakoff, W., "Erosion Prediction in Turbomachinery Resulting from Environmental Solid Particles," Journal of Aircraft, Vol. 12, 1975, pp. 471-547.

[31] Sommerfeld, M., "Modeling of Particle-Wall Collisions in Confined Gas-Particle Flows," International Journal of Multiphase Flow, Vol. 18, 1992, pp. 905-926.

[32] Sommerfeld, M., "Particle-Wall Collisions: Experimental Studies and Numerical Models," ASME FED, Vol. 166, 1993, pp. 183-191.

[33] Selig, M. S., "Multipoint Inverse Airfoil Design Method Based on Conformal Mapping," AIAA Journal, Vol. 30, No. 5, May 1992.

[34] Saeed, F., and Selig, M. S., "A Multipoint Inverse Airfoil Design Method for Slot-Suction Airfoils," Journal of Aircraft, Vol. 33, No. 5, July-Aug. 1996. 


\section{IMPORTANT: PLEASE READ CAREFULLY.}

\section{Queries}

When production of AIAA journal papers begins, the official approved PDF is considered the authoritative manuscript. Authors are asked to submit source files that match the PDF exactly, to ensure that the final published article is the version that was reviewed and accepted by the associate editor. Once a paper has been accepted, any substantial corrections or changes must be approved by the associate editor before they can be incorporated.

If you and the EIC settled on some final changes to your manuscript after it was accepted, it is possible that your page proofs do not reflect these final changes. If that is the case, please submit these changes as itemized corrections to the proofs.

If final changes were made to the figures, please check the figures appearing in the proofs carefully. While it is usual procedure to use the figures that exist in the source file, if discrepancies are found between figures (manuscript source file vs the approved PDF), the figures from the PDF are inserted in the page proofs, again deferring to the PDF as the authoritative manuscript. If you find that agreed-upon final changes to your figures are not appearing in your page proofs, please let us know immediately.

Q1. Your vectors arrows have been removed and the characters made bold italic, per AIAA style.

Q2. You say here "engine operational envelop." Do you mean "envelope"?

Q3. Please check this notation of million.

Q4. Does CANICE and LEWICE need to be defined for the reader?

Q5. Is this a permanent website that can be accessed indefinitely? If not permanent, it will need to be put into the text as a footnote.

Q6. Please give the city for the publisher in [7].

Q7. Can you please give page numbers for [10]?

Q8. Can you please give an issue number or month for [18, 19, 23-25, 30-32]?

Q9. Please spell out the journal title in [32].

Q10. Please give the page numbers for $[33,34]$. 